\title{
The Maximum Number of Odd Integral Distances Between Points in the Plane
}

\author{
L. Piepmeyer* \\ Technische Universität Braunschweig, Diskrete Mathematik, \\ Pockelsstrasse 14, D-38106 Braunschweig, Germany
}

Dedicated to Professor Dr. H.-J. Kanold on the occasion of his eightieth birthday

\begin{abstract}
Four points in the plane with pairwise odd integral distances do not exist. The maximum number of odd distances between $n$ points in the plane is proved to be $n^{2} / 3+r(r-3) / 6$ for all $n$, where $r=1,2,3$ and $n \equiv r(\bmod 3)$. This solves a recently stated problem of Erdôs.
\end{abstract}

For every dimension $d$ and for every natural number $n$ there exist $n$ points in the Euclidean space $E^{d}$ with pairwise integral distances, since $n$ equidistributed points on a line determine by an appropriate scaling only integral distances. However, the maximum number of points with pairwise odd integral distances in $E^{d}$ is $d+2$ if $d+2 \equiv 0$ $(\bmod 16)$ and $d+1$ in the remaining cases (see [2]; an independent proof was given in [4]). Thus four points in the plane with mutual odd distances do not exist.

Erdős [1] asked for the maximum number $f(n)$ of odd integral distances occurring between $n$ points in the plane. This maximum $f(n)$ is determined in Theorem 1 . The proof is based on a construction given in [3]. Moreover, we make use of Turán's theorem from graph theory (see p. 30 of [5]).

Turán's Theorem. The maximum number of edges in a graph with $n$ vertices that does not contain a complete subgraph with four vertices is

$$
\frac{n^{2}}{3}+\frac{r(r-3)}{6} \quad \text { with } r=1,2 \text {, or } 3 \text {, and } n \equiv r(\bmod 3) \text {. }
$$

The upper bound is attained if and only if the graph is isomorphic to the complete 3-partite graph $T_{n}$ with three vertex classes that have almost the same size.

\footnotetext{
* Current address: Morassistrasse 24, 80469 Munich, Germany. lotharp@informix.com.
} 
Theorem 1. The maximum number $f(n)$ of odd integral distances between $n$ points in the plane is

$$
f(n)=\frac{n^{2}}{3}+\frac{r(r-3)}{6} \quad \text { with } \quad r=1,2, \text { or } 3, \text { and } n \equiv r \quad(\bmod 3)
$$

Proof. The upper bound $f(n) \leq n^{2} / 3+r(r-3) / 6$ follows immediately from Turán's theorem if we consider a set $\mathcal{S}$ of $n$ points in the plane with the maximum number of odd integral distances together with the graph $G(\mathcal{S})$ induced by the points and the odd distances of $\mathcal{S}$. Since $G(\mathcal{S})$ does not contain a complete graph with four vertices the number of edges in $G(\mathcal{S})$ and hence the upper bound for the number of odd integral distances in $\mathcal{S}$ follows by Turán's theorem.

The lower bound $f(n) \geq n^{2} / 3+r(r-3) / 6$ follows by construction of a set $\mathcal{P}_{m}=$ $\left\{p_{0}, \ldots, p_{3 m-1}\right\}$ of $n=3 m$ points in the complex plane with $3 m^{2}$ odd integral distances for each natural number $m$. Since $G\left(\mathcal{P}_{m}\right)$ is isomorphic to $T_{3 m}$ by Turán's theorem, the deletion of one or two appropriate vertices leaves a $T_{3 m-1}$ or a $T_{3 m-2}$ so that the corresponding point sets prove the lower bound for $n=3 m-1$ or $n=3 m-2$.

For the third root of unity $\rho=(-1+\sqrt{3} i) / 2$ with $i^{2}=-1$, and for $\omega=3+\rho$ let $\eta_{k}=7^{m-\lfloor k / 3\rfloor} \rho^{k} \omega^{2\lfloor k / 3\rfloor}$, and $p_{k}=\sqrt{3} \eta_{k}^{2} /\left(3 \cdot 7^{m}\right)$ with $0 \leq k<3 m$. This is the special case $R=7^{m}=(\omega \bar{\omega})^{m}$ of a construction given in [3] (see Fig. 1 for the case $m=2$ ). The points of the construction consist of the vertices of a regular triangle of side lengths $7^{m}$ that is rotated $m-1$ times about its center through an angle $\theta=\arccos 71 / 98$.

In [3] it is shown that $\bar{\eta}_{s} \eta_{t}=x+\rho 7^{m}\left|p_{s}-p_{t}\right|$ for $0 \leq s<t<3 m$ and with a real number $x$. Comparing the imaginary parts implies

$$
\left|p_{s}-p_{t}\right|=\frac{\operatorname{Im}\left(\bar{\eta}_{s} \eta_{t}\right)}{7^{m} \operatorname{Im}(\rho)}=\frac{(2 \sqrt{3} / 3) \operatorname{Im}\left(\bar{\eta}_{s} \eta_{t}\right)}{7^{m}} .
$$

With $\bar{\rho}=\rho^{2}$ and $\omega \bar{\omega}=7$ it follows that

$$
\bar{\eta}_{s} \eta_{t}=7^{2 m+\lfloor s / 3\rfloor-\lfloor t / 3\rfloor} \rho^{t+2 s} \omega^{2\lfloor t / 3\rfloor-2\lfloor s / 3\rfloor} .
$$

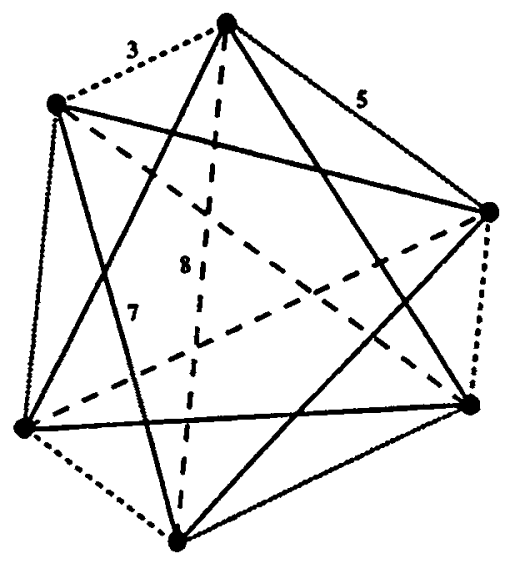

Fig. 1. Six points with 12 odd distances. 
Since all occurring distances $\left|p_{s}-p_{t}\right|$ are integers as shown in [3] the number of odd distances equals the number of odd numbers $(2 \sqrt{3} / 3) \operatorname{Im}\left(\rho^{t+2 s} \omega^{2\lfloor t / 3\rfloor-2\lfloor s / 3\rfloor}\right)$, with $0 \leq s<t<3 m$.

With $\omega^{2}=8+5 \rho, \rho^{2}=-1-\rho$, and $\rho^{3}=1$, and with

$$
\begin{aligned}
(8+5 \rho)^{k}= & \sum_{\nu \equiv 0(\bmod 3)} 8^{k-v} 5^{v}\left(\begin{array}{l}
k \\
v
\end{array}\right)+\rho \sum_{\nu \equiv 1(\bmod 3)} 8^{k-v} 5^{v}\left(\begin{array}{l}
k \\
v
\end{array}\right) \\
& -(1+\rho) \sum_{v \equiv 2(\bmod 3)} 8^{k-v} 5^{v}\left(\begin{array}{l}
k \\
v
\end{array}\right)
\end{aligned}
$$

it follows that $\omega^{2 k}$ and $\rho^{k}$ are both of the form $a+b \rho$ with integers $a, b$ satisfying $a \equiv 1,0,1(\bmod 2)$ and $b \equiv 0,1,1(\bmod 2)$ for $k \equiv 0,1,2(\bmod 3)$, respectively. Thus $(2 \sqrt{3} / 3) \operatorname{Im}\left(\omega^{2 k} \rho^{l}\right)$ for fixed $k$ is an odd number in exactly two of the three cases $1=0,1,2$. Now it follows for all pairs $s, t$ with $0 \leq\lfloor s / 3\rfloor<\lfloor t / 3\rfloor<m$ that exactly six of all nine distances $\left|p_{s}-p_{t}\right|$ are odd. In the remaining $m$ cases where $\lfloor s / 3\rfloor=\lfloor t / 3\rfloor$ all three distances $\left|p_{s}-p_{t}\right|$ are odd. Altogether there are $6\left(\begin{array}{c}m \\ 2\end{array}\right)+3 m=3 m^{2}$ odd integral distances in $\mathcal{P}_{m}$ and the proof is complete.

\section{References}

1. P. Erdős, Oral communication, Conference on Graph Theory, Combinatorics, and Computation, 1994, Boca Raton, FL.

2. R. L. Graham, B. L. Rothschild, and E. G. Straus, Are there $n+2$ points in $E^{n}$ with pairwise odd integral distances? Amer. Math. Monthly 81 (1974), 21-25.

3. H. Harborth, A. Kemnitz, and M. Möller, An upper bound for the minimum diameter of integral point sets, Discrete Comput. Geom. 9 (1993), 427-432.

4. M. Rosenfield, Oral communication, Conference on Graph Theory, Combinatorics, and Computation, 1994, Boca Raton, FL.

5. J. H. van Lint and R. M. Wilson, A Course in Combinatorics, Cambridge University Press, Cambridge, 1992.

Received February 27, 1995, and in revised form August 31, 1995. 